\title{
Black Holes: A General Introduction
}

\author{
Jean-Pierre Luminet \\ Observatoire de Paris-Meudon, Département d'Astrophysique Relativiste et de \\ Cosmologie, CNRS UPR-176, F-92195 Meudon Cedex, France
}

\begin{abstract}
Our understanding of space and time is probed to its depths by black holes. These objects, which appear as a natural consequence of general relativity, provide a powerful analytical tool able to examine macroscopic and microscopic properties of the universe. This introductory article presents in a pictorial way the basic concepts of black hole's theory, as well as a description of the astronomical sites where black holes are suspected to lie, namely binary $\mathrm{X}$-ray sources and galactic nuclei.
\end{abstract}

\section{The Black Hole Mystery}

Let me begin with an old Persian story. Once upon a time, the butterflies organized a summer school devoted to the great mystery of the flame. Many discussed about models but nobody could convincingly explain the puzzle. Then a bold butterfly enlisted as a volunteer to get a real experience with the flame. He flew off to the closest castle, passed in front of a window and saw the light of a candle. He went back, very excited, and told what he had seen. But the wise butterfly who was the chair of the conference said that they had no more information than before. Next, a second butterfly flew off to the castle, crossed the window and touched the flame with his wings. He hardly came back and told his story; the wise chairbutterfly said "your explanation is no more satisfactory". Then a third butterfly went to the castle, hit the candle and burned himself into the flame. The wise butterfly, who had observed the action, said to the others: "Well, our friend has learned everything about the flame. But only him can know, and that's all".

As you can guess, this story can easily be transposed from butterflies to scientists confronted with the mystery of black holes. Some astronomers, equipped with powerful instruments such as orbiting telescopes, make very distant and indirect observations on black holes; like the first butterfly, they acknowledge the real existence of black holes but they gain very little information on their real nature. Next, theoretical physicists try to penetrate more deeply into the black hole mystery by using tools such as general relativity, quantum mechanics and higher mathematics; like the second butterfly, they get a little bit more information, but not so much. The equivalent of the third butterfly would be a spationaut plunging directly into a black hole, but eventually he will not be able to go back and tell his story. Nevertheless, by using numerical calculations such as those performed at the Observatoire de Meudon I will show you later, outsiders can get some idea of what happens inside a black hole. 


\section{Physics of Black Holes}

\section{$2.1 \quad$ Light imprisoned}

Let us begin to play like the second butterfly, and explore the black hole from the point of view of theoretical physics. An elementary definition of a black hole is a region of space-time in which the gravitational potential, $G M / R$, exceeds the square of the speed of light, $c^{2}$. Such a statement has the merit to be independent of the details of gravitational theories. It can be used in the framework of Newtonian theory. It also provides a more popular definition of a black hole, according to which any astronomical body whose escape velocity exceeds the speed of light must be a black hole. Indeed, such a reasoning was done two centuries ago by John Michell and Pierre-Simon de Laplace. In the Philosophical Transactions of the Royal Society (1784), John Michell pointed out that "if the semi diameter of a sphere of the same density with the sun were to exceed that of the sun in the proportion of 500 to $1,(.$.$) all light emitted from such a body$ would be made to return towards it", and independently, in 1796, Laplace wrote in his Exposition du Système du Monde: "Un astre lumineux de même densité que la terre et dont le diamètre serait deux cents cinquante fois plus grand que celui du soleil, ne laisserait, en vertu de son attraction, parvenir aucun de ses rayons jusqu'à nous ; il est donc possible que les plus grands corps lumineux de l'univers soient, par cela même, invisibles". Since the density imagined at this time was that of ordinary matter, the size and the mass of the associated "invisible body" were huge - around $10^{7}$ solar masses, corresponding to what is called today a "supermassive" black hole. Nevertheless, from the numerical figures first proposed by Michell and Laplace, one can recognize the well-known basic formula giving the critical radius of a body of mass $M$ :

$$
R_{S}=\frac{2 G M}{c^{2}} \approx 3 \frac{M}{M_{\odot}} \mathrm{km},
$$

where $M_{\odot}$ is the solar mass. Any spherical body of mass $M$ confined within the critical radius $R_{S}$ must be a black hole.

These original speculations were quickly forgotten, mainly due to the development of the wave theory of light, within the framework of which no calculation

of the action of gravitation on light propagation was performed. The advent of general relativity, a fully relativistic theory of gravity in which light is submitted to gravity, gave rise to new speculations and much deeper insight into black holes.

To pictorially describe black holes in space-time, I shall use light cones. Let me recall what a light cone is. In figure 1, a luminous flash is emitted at a given point of space. The wavefront is a sphere expanding at a velocity of $c=300000 \mathrm{~km} / \mathrm{s}$, shown in a) at three successive instants. The light cone representation in b) tells the complete story of the wavefront in a single spacetime diagram. As one space dimension is removed, the spheres become circles. The expanding circles of light generate a cone originating at the emission point. 
If, in this diagram, we choose the unit of length as $300000 \mathrm{~km}$ and the unit of time as 1 second, all the light rays travel at $45^{\circ}$.

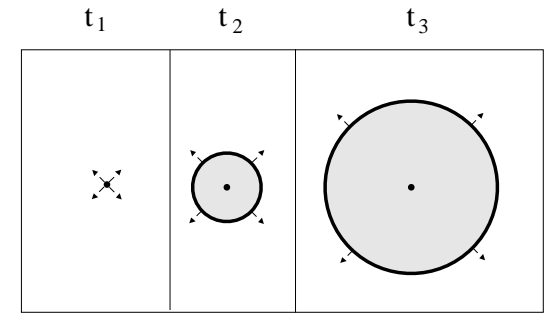

(a) Spatial representation

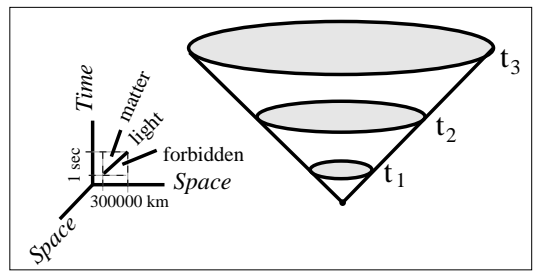

(b) The light cone

Figure 1

Fig. 1 The light cone.

The light cone allows us to depict the causal structure of any space-time. Take for instance the Minkowski flat space-time used in Special Relativity (figure 2). At any event $E$ of space-time, light rays generate two cones (shaded zone). The rays emitted from $E$ span the future light cone, those received in $E$ span the past light cone. Physical particles cannot travel faster than light: their trajectories remain confined within the light cones. No light ray or particle which passes

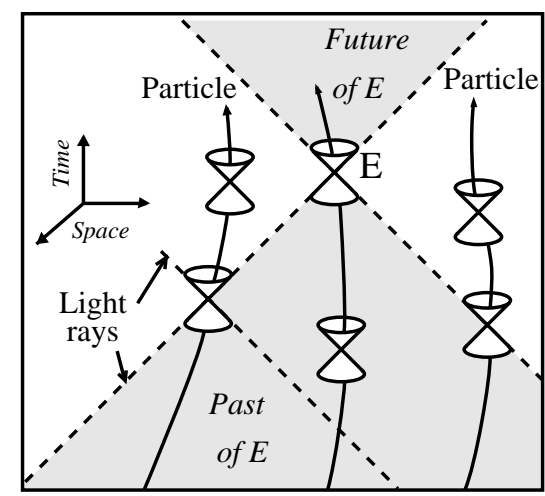

Figure 2

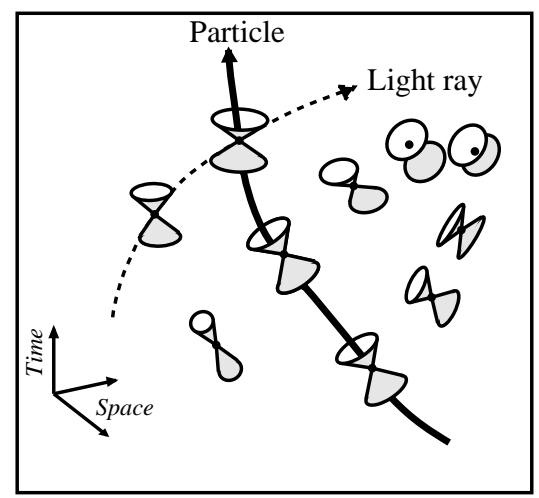

Figure 3

Fig. 2\&3 The space-time continuum of Special Relativity and the soft space-time of General Relativity. 
through $E$ is able to penetrate the clear zone. The invariance of the speed of light in vacuum is reflected by the fact that all the cones have the same slope. This is because the space-time continuum of Special Relativity, free from gravitating matter, is flat and rigid. As soon as gravity is present, space-time is curved and Special Relativity leaves room to General Relativity. Since the Equivalence Principle states the influence of gravity on all types of energy, the light cones follow the curvature of space-time (figure 3). They bend and deform themselves according to the curvature. Special Relativity remains locally valid however: the worldlines of material particles remain confined within the light cones, even when the latter are strongly tilted and distorted by gravity.

\subsection{Spherical collapse}

Let us now examine the causal structure of space-time around a gravitationally collapsing star - a process which is believed to lead to black hole formation. Figure 4 shows the complete history of the collapse of a spherical star, from its initial contraction until the formation of a black hole and a singularity. Two space dimensions are measured horizontally, and time is on the vertical axis, measured upwards. The centre of the star is at $r=0$. The curvature of spacetime is visualized by means of the light cones generated by the trajectories of light rays. Far away from the central gravitational field, the curvature is so weak that the light cones remain straight. Near the gravitational field, the cones are distorted and tilted inwards by the curvature. On the critical surface of radius $r=2 M$, the cones are tipped over at $45^{\circ}$ and one of their generators becomes vertical, so that the allowed directions of propagation of particles and electromagnetic waves are oriented towards the interior of this surface. This is the event horizon, the boundary of the black hole (grey region). Beyond this, the stellar matter continues to collapse into a singularity of zero volume and infinite density at $r=0$. Once a black hole has formed, and after all the stellar matter has disappeared into the singularity, the geometry of space-time itself continues to collapse towards the singularity, as shown by the light cones.

The emission of the light rays at $E_{1}, E_{2}, E_{3}$ and $E_{4}$ and their reception by a distant astronomer at $R_{1}, R_{2}, R_{3}, \ldots$ well illustrate the difference between the proper time, as measured by a clock placed on the surface of the star, and the apparent time, measured by an independent and distant clock. The (proper) time interval between the four emission events are equal. However, the corresponding reception intervals become longer and longer. At the limit, light ray emitted from $E_{4}$, just when the event horizon is forming, takes an infinite time to reach the distant astronomer. This phenomenon of "frozen time" is just an illustration of the extreme elasticity of time predicted by Einstein's relativity, according to which time runs differently for two observers with a relative acceleration - or, from the Equivalence Principle, in different gravitational potentials. A striking consequence is that any outer astronomer will never be able to see the formation of a black hole. The figure 5 shows a picturesque illustration of frozen time. A spaceship has the mission of exploring the interior of a black hole - preferably a 


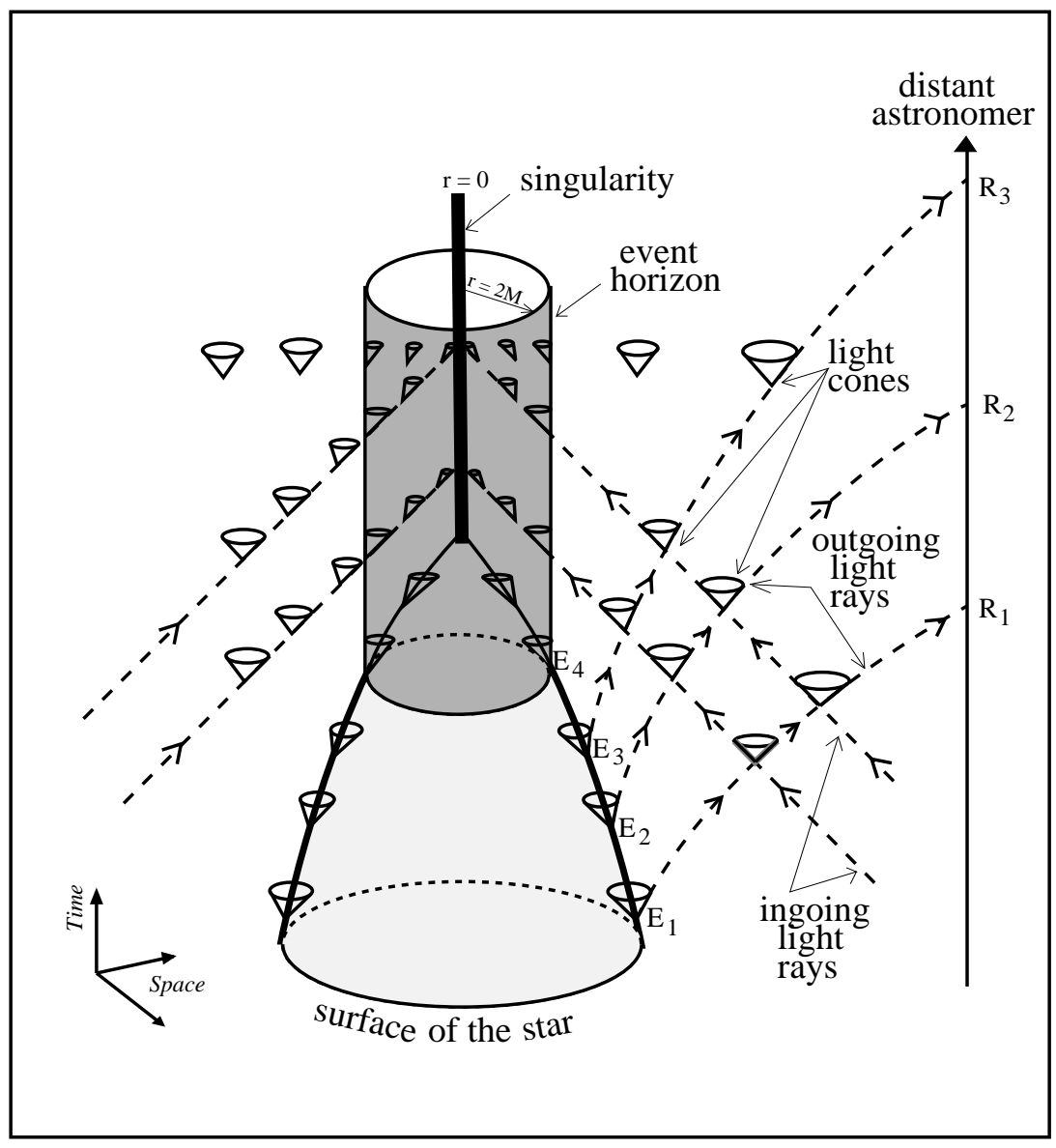

Figure 4

Fig. 4 A space-time diagram showing the formation of a black hole by gravitational collapse.

big one, so that it is not destroyed too quickly by the tidal forces. On board the ship, the commander sends a solemn salute to mankind, just at the moment when the ship crosses the horizon. His gesture is transmitted to distant spectators via television. The film on the left shows the scene on board the spaceship in proper time, that is, as measured by the ship's clock as the ship falls into the black hole. The astronaut's salute is decomposed into instants at proper time intervals of 0.2 second. Crossing of the event horizon (black holes have not a hard surface) is not accompanied by any particular event. The film on the right shows the scene received by distant spectators via television. It is also decomposed into intervals 
of apparent time of 0.2 second. At the beginning of his gesture, the salute is slightly slower than the real salute, but initially the delay is too small to be noticed, so the films are practically identical. It is only very close to the horizon that apparent time starts suddenly to freeze; the film on the right then shows the astronaut eternally frozen in the middle of his salute, imperceptibly reaching the limiting position where he crossed the horizon. Besides this effect, the shift in the frequencies in the gravitational field (the so-called Einstein's effect) causes the images to weaken, and they soon become invisible.

All these effects follow rather straightforwardly from equations. In General Relativity, the vacuum space-time around a spherically symmetric body is described by the Schwarzschild metric:

$$
d s^{2}=-\left(1-\frac{2 M}{r}\right) d t^{2}+\left(1-\frac{2 M}{r}\right)^{-1} d r^{2}+r^{2} d \Omega^{2},
$$

where $d \Omega^{2}=d \theta^{2}+\sin ^{2} \theta d \phi^{2}$ is the metric of a unit 2-sphere, and we have set the gravity's constant $G$ and the speed of light $c$ equal to unity. The solution describes the external gravitational field generated by any static spherical mass, whatever its radius (Birkhoff's theorem, 1923).

When the radius is greater than $2 M$, there exists "interior solutions" depending on the equation of state of the stellar matter, which are non-singular at $r=0$ and that match the exterior solution. However, as soon as the body is collapsed under its critical radius $2 M$, the Schwarzschild metric is the unique solution for the gravitational field generated by a spherical black hole. The event horizon, a sphere of radius $r=2 M$, is a coordinate singularity which can be removed by a suitable coordinate transformation (see below). There is a true gravitational singularity at $r=0$ (in the sense that some curvature components diverge) that cannot be removed by any coordinate transformation. Indeed the singularity does not belong to the space-time manifold itself. Inside the event horizon, the radial coordinate $r$ becomes timelike. Hence every particle that crosses the event horizon is unavoidably catched by the central singularity. For radial free-fall along a trajectory with $r \rightarrow 0$, the proper time (as measured by a comoving clock) is given by

$$
\tau=\tau_{0}-\frac{4 M}{3}\left(\frac{r}{2 M}\right)^{3 / 2}
$$

and is well-behaved at the event horizon. The apparent time (as measured by a distant observer) is given by

$$
t=\tau-4 M\left(\frac{r}{2 M}\right)^{1 / 2}+2 M \ln \frac{\sqrt{r / 2 M}+1}{\sqrt{r / 2 M}-1}
$$

and diverges to infinity as $r \rightarrow 2 M$, see figure 6 .

The Schwarzschild coordinates, which cover only $2 M \leq r<\infty,-\infty<t<$ $+\infty$, are not well adapted to the analysis of the causal structure of space-time 


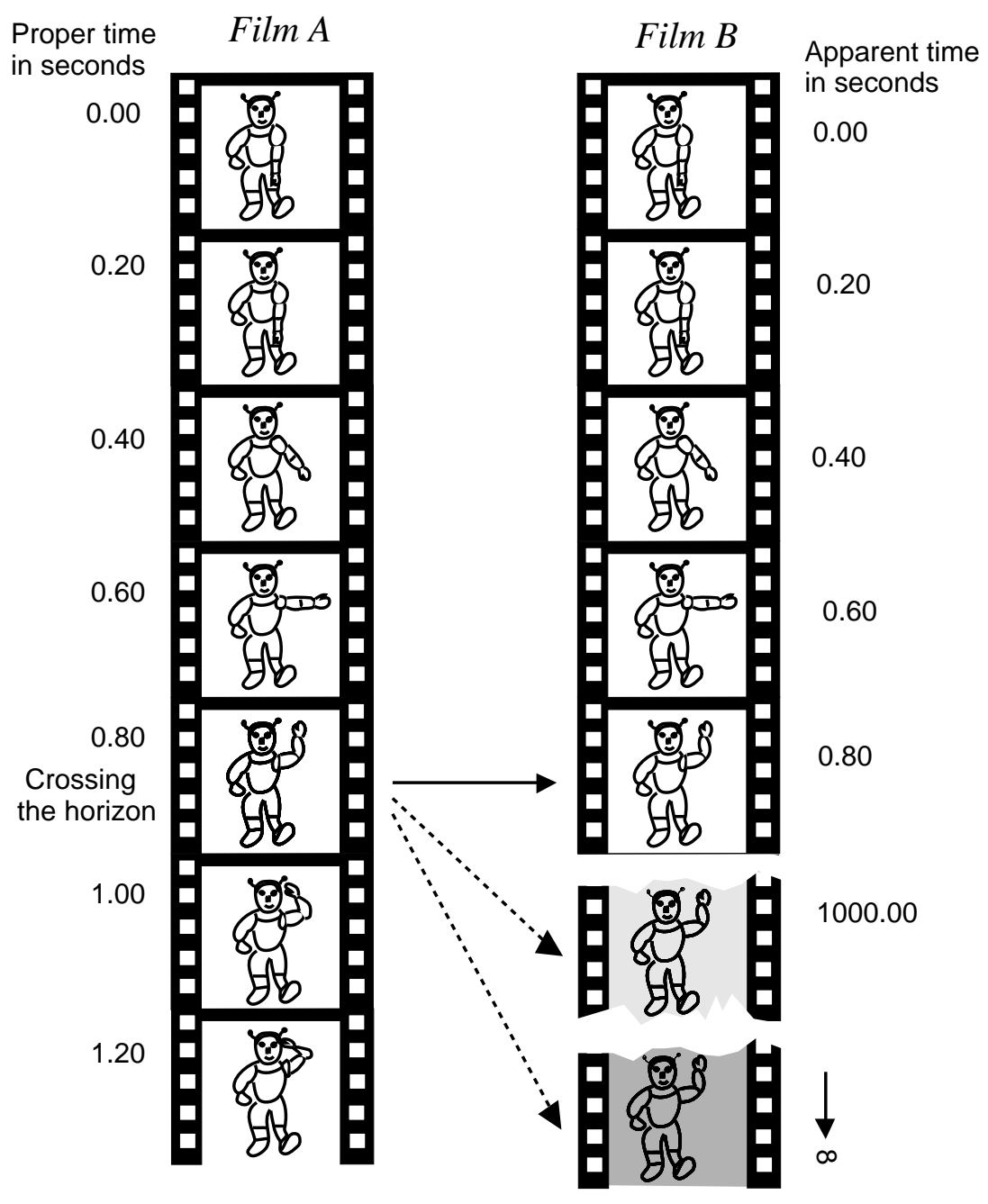

Figure 5

Fig. 5 The astronauts salute.

near the horizon, because the light cones, given by $d r= \pm\left(1-\frac{2 M}{r}\right) d t$, are not defined on the event horizon. We better use the so-called Eddington-Finkelstein coordinates - indeed discovered by Lemaitre in 1933 but remained unnoticed. Introducing the "ingoing" coordinate

$$
v=t+r+2 M \ln \left(\frac{r}{2 M}-1\right)
$$




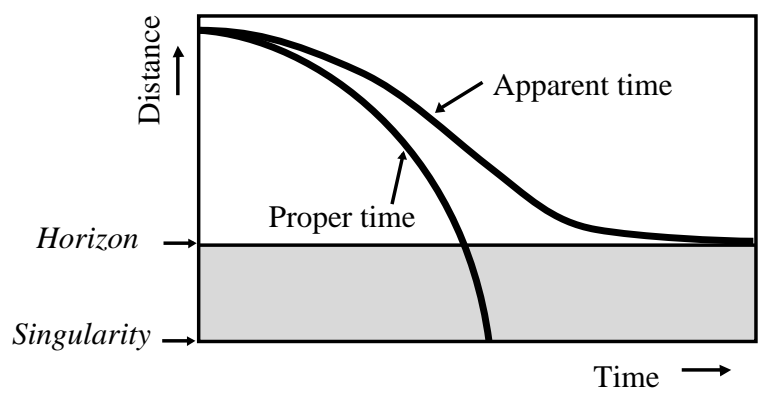

Figure 6

Fig. 6 The two times of a black hole.

the Schwarzschild metric becomes

$$
d s^{2}=-\left(1-\frac{2 M}{r}\right) d v^{2}+2 d v d r+r^{2} d \Omega^{2} .
$$

Now the light cones are perfectly well behaved. The ingoing light rays are given by

$$
d v=0
$$

the outgoing light rays by

$$
d v=\frac{2 d r}{1-\frac{2 M}{r}} .
$$

The metric can be analytically continued to all $r>0$ and is no more singular at $r=2 M$. Indeed, in figure 4 such a coordinate system was already used.

\subsection{Non spherical collapse}

A black hole may well form from an asymmetric gravitational collapse. However the deformations of the event horizon are quickly dissipated as gravitational radiation; the event horizon vibrates according to the so-called "quasi-normal modes" and the black hole settles down into a final axisymmetric equilibrium configuration.

The deepest physical property of black holes is that asymptotic equilibrium solutions depend only on three parameters: the mass, the electric charge and the angular momentum. All the details of the infalling matter other than mass, electric charge and angular momentum are washed out. The proof followed from efforts over 15 years by half a dozen of theoreticians, but it was originally suggested as a conjecture by John Wheeler, who used the picturesque formulation "a black hole has no hair". Markus Heusler's lectures in this volume will develop this so-called "uniqueness theorem". 


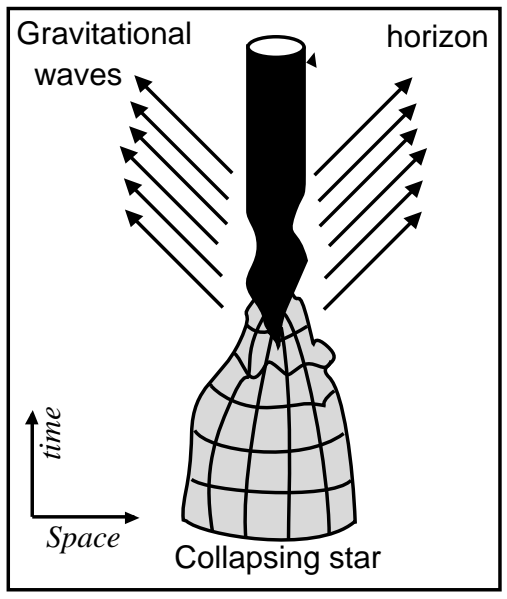

Figure 7

Fig. 7 Gravitational collapse of a star.

As a consequence, there exists only 4 exact solutions of Einstein's equations describing black hole solutions with or without charge and angular momentum:

- The Schwarzschild solution (1917) has only mass $M$; it is static, spherically symmetric.

- The Reissner-Nordström solution (1918), static, spherically symmetric, depends on mass $M$ and electric charge $Q$.

- The Kerr solution (1963), stationary, axisymmetric, depends on mass and angular momentum.

- The Kerr-Newman solution (1965), stationary and axisymmetric, depends on all three parameters $M, J, Q$.

The 3-parameters Kerr-Newman family is the most general solution, corresponding to the final state of black hole equilibrium. In Boyer-Lindquist coordinates, the Kerr-Newman metric is given by

$$
\begin{aligned}
& d s^{2}=-\left(1-\frac{2 M r}{\Sigma}\right) d t^{2}-4 M r a \frac{\sin ^{2} \theta}{\Sigma} d t d \phi \\
& +\left(r^{2}+a^{2}+\frac{2 M r a^{2} \sin ^{2} \theta}{\Sigma}\right) \sin ^{2} \theta d \phi^{2}+\frac{\Sigma}{\Delta} d r^{2}+\Sigma d \theta^{2}
\end{aligned}
$$

where $\Delta \equiv r^{2}-2 M r+a^{2}+Q^{2}, \Sigma \equiv r^{2}+a^{2} \cos ^{2} \theta, a \equiv J / M$ is the angular momentum per unit mass. The event horizon is located at distance $r_{+}=M+$ $\sqrt{M^{2}-Q^{2}-a^{2}}$. 
From this formula we can see, however, that the black hole parameters cannot be arbitrary. Electric charge and angular momentum cannot exceed values corresponding to the disappearance of the event horizon. The following constraint must be satisfied: $a^{2}+Q^{2} \leq M^{2}$.

When the condition is violated, the event horizon disappears and the solution describes a naked singularity instead of a black hole. Such odd things should not exist in the real universe (this is the statement of the so-called Cosmic Censorship Conjecture, not yet rigorously proved). For instance, for uncharged rotating configuration, the condition $J_{\max }=M^{2}$ corresponds to the vanishing of surface gravity on the event horizon, due to "centrifugal forces"; the corresponding solution is called extremal Kerr Solution. Also, the maximal allowable electric charge is $Q_{\max }=M \approx 10^{40} e M / M_{\odot}$, where $e$ is the electron charge, but it is to be noticed that in realistic situations, black holes should not be significantly charged. This is due to the extreme weakness of gravitational interaction compared to electromagnetic interaction. Suppose a black hole forms with initial positive charge $Q_{i}$ of order $M$. In realistic conditions, the black hole is not isolated in empty space but is surrounded by charged particles of the interstellar medium, e.g. protons and electrons. The black hole will predominantly attract electrons and repel protons with charge $e$ by its electromagnetic field, and predominantly attract protons of mass $m_{p}$ by its gravitational field. The repulsive electrostatic force on protons is larger than the gravitational pull by the factor $e Q / m_{p} M \approx e / m_{p} \approx 10^{18}$. Therefore, the black hole will neutralize itself almost instantaneously. As a consequence, the Kerr solution, obtained in equation (9) by putting $Q=0$, can be used for any astrophysical purpose involving black holes. It is also a good approximation to the metric of a (not collapsed) rotating star at large distance, but it has not been matched to any known solution that could represent the interior of a star.

The Kerr metric in Boyer-Lindquist coordinates has singularities on the axis of symmetry $\theta=0$ - obviously a coordinate singularity - and for $\Delta=0$. One can write $\Delta=\left(r-r_{+}\right)\left(r-r_{-}\right)$with $r_{+}=M+\sqrt{M^{2}-a^{2}}$. The distance $r_{+}$ defines the outer event horizon (the surface of the rotating black hole), whereas $r_{-}$defines the inner event horizon. Like in Schwarzschild metric (where $r_{+}$and $r_{-}$coincide at the value $2 M$ ), the singularities at $r=r_{+}, r=r_{-}$are coordinate singularities which can be removed by a suitable transformation analogous to the ingoing Eddington-Finkelstein coordinates for Schwarzschild space-time. For full mathematical developments of Kerr black holes, see Chandrasekhar (1992) and O'Neill (1995).

\subsection{The black hole maelstrom}

There is a deep analogy between a rotating black hole and the familiar phenomenon of a vortex - such as a giant maelstrom produced by sea currents. If we cut a light cone at fixed time (a horizontal plane in figure 8), the resulting spatial section is a "navigation ellipse" which determines the limits of the permitted trajectories. If the cone tips over sufficiently in the gravitational field, 


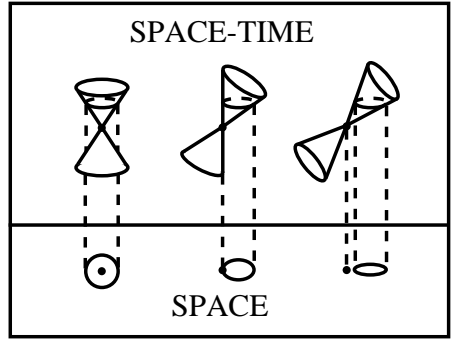

Figure 8

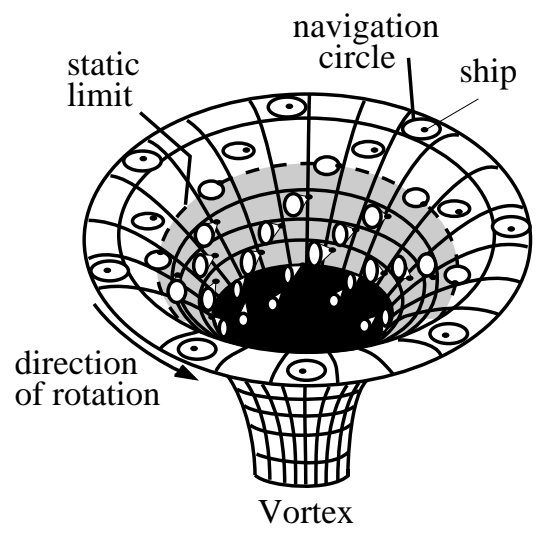

Figure 9

Fig. 8\&9 Navigation circles in the black hole maelstrom.

the navigation ellipse detaches itself from the point of emission. The permitted trajectories are confined within the angle formed by the tangents of the circle, and it is impossible to go backwards.

This projection technique is useful to depict the causal structure of spacetime around a rotating black hole (figure 9). The gravitational well caused by a rotating black hole resembles a cosmic maelstrom. A spaceship travelling in the vicinity is sucked towards the centre of the vortex like a boat. In the region outside the so-called static limit (clear), it can navigate to whereever it wants. In the zone (in grey) comprised between the static limit and the event horizon, it is forced to rotate in the same direction as the black hole; its ability to navigate freely is decreased as it is sucked inwards, but it can still escape by travelling in an outwards spiral. The dark zone represents the region inside the event horizon: any ship which ventured there would be unable to escape even if it was travelling at the speed of light. A fair illustration is the Edgar Poe's short story: A descent into the maelstrom (1840).

The static limit is a hypersurface of revolution, given by the equation $r=$ $M+\sqrt{M^{2}-a^{2} \cos ^{2} \theta}$. As we can see from figure 10, it intersects the event horizon at its poles $\theta=0, \pi$ but it lies outside the horizon for other values of $\theta$. The region between the static limit and the event horizon is called ergoregion. There, all stationary observers must orbit the black hole with positive angular velocity. The ergoregion contains orbits with negative energy. Such a property has lead to the idea of energy extraction from a rotating black hole. Roger Penrose (1969) suggested the following mechanism. A distant experimentalist fires a projectile in the direction of the ergosphere along a suitable trajectory (figure 10). When it arrives the projectile splits into two pieces: one of them is captured by the black hole along a retrograde orbit, while the other flies out of the ergosphere 


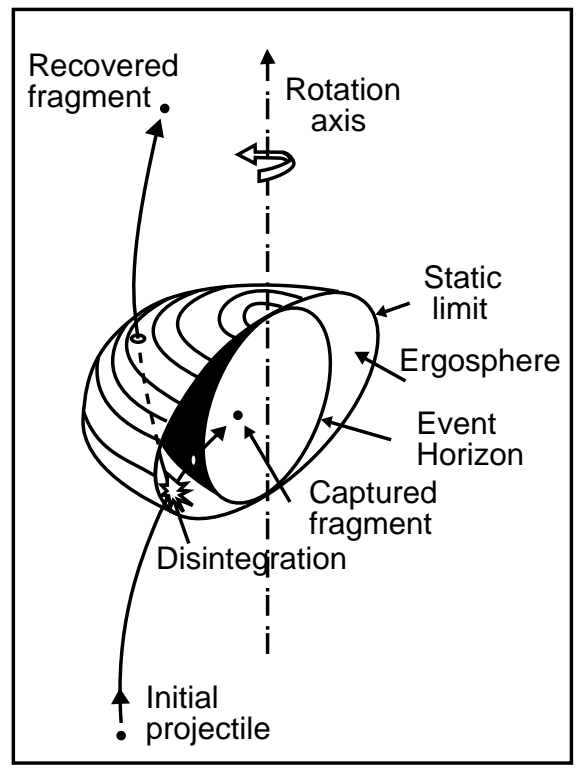

Figure 10

Fig. 10 Cross-section of a rotating black hole.

and is recovered by the experimentalist. Penrose has demonstrated that the experimentalist could direct the projectile in such a way that the returning piece has a greater energy than that of the initial projectile. This is possible if the fragment captured by the black hole is travelling in a suitable retrograde orbit (that is orbiting in the opposite sense to the rotation of the black hole), so that when it penetrates the black hole it slightly reduces the hole's angular momentum. The net result is that the black hole looses some of its rotational energy and the difference is carried away by the escaping fragment.

The amount of energy that can theoretically be extracted from a black hole has been calculated by Christodolou and Ruffini (1971). The total mass-energy of a black hole is

$$
M^{2}=\frac{J^{2}}{4 M_{i r}^{2}}+\left(\frac{Q^{2}}{4 M_{i r}}+M_{i r}\right)^{2}
$$

where $M_{i r} \equiv \frac{1}{2} \sqrt{\left(M+\sqrt{M^{2}-Q^{2}-a^{2}}\right)^{2}+a^{2}}$. The first term corresponds to the rotational energy, the second one to the Coulomb energy, the third one to an "irreducible" energy. The rotational energy and the Coulomb energy are extractable by physical means such as the Penrose process, the superradiance (analogous to stimulated emission in atomic physics) or electrodynamical processes (see Norbert Straumann's lectures for details), while the irreducible part 
cannot be lowered by classical (e.g. non quantum) processes. The maximum extractable energy is as high a 29 per cent for rotational energy and 50 per cent for Coulomb energy. It is much more efficient that, for instance, nuclear energy release (0.7 per cent for hydrogen burning).

\subsection{Black hole thermodynamics}

It is interesting to mention that the irreducible mass is related to the area $A$ of the event horizon by $M_{i r}=\sqrt{A / 16 \pi}$. Therefore the area of an event horizon cannot decrease in time by any classical process. This was first noticed by Stephen Hawking, who drew the striking analogy with ordinary thermodynamics, in which the entropy of a system never decreases in time. Such a property has motivated a great deal of theoretical efforts in the 1970's to better understand the laws of black hole dynamics - i.e. the laws giving the infinitesimal variations of mass, area and other black hole quantities when a black hole interacts with the external universe - and to push the analogy with thermodynamical laws. For the development of black hole thermodynamics, see G. Neugebaueur's and W. Israel's lectures in this volume. Let me just recall that black hole mechanics is governed by four laws which mimic classical thermodynamics:

- Zeroth law.

In thermodynamics: all parts of a system at thermodynamical equilibrium have equal temperature $T$.

In black hole mechanics: all parts of the event horizon of a black hole at equilibrium have the same surface gravity $g$. The surface gravity is given by the Smarr's formula $M=g A / 4 \pi+2 \Omega_{H} J+\Phi_{H} Q$, where $\Omega_{H}$ is the angular velocity at the horizon and $\Phi_{H}$ is the co-rotating electric potential on the horizon. This is a quite remarkable property when one compares to ordinary astronomical bodies, for which the surface gravity depends on the latitude. Whatever a black hole is flattened by centrifugal forces, the surface gravity is the same at every point.

- First Law.

In thermodynamics: the infinitesimal variation of the internal energy of a system with temperature $T$ at pressure $P$ is related to the variation of entropy $d S$ and the variation of pressure $d P$ by $d U=T d S-P d V$.

In black hole dynamics: the infinitesimal variations of the mass $M$, the charge $Q$ and the angular momentum $J$ of a perturbed stationary black hole are related by $d M=\frac{g}{8 \pi} d A+\Omega_{H} d J+\Phi_{H} d Q$.

- Second Law.

In thermodynamics, entropy can never decrease: $d S \geq 0$.

In black hole dynamics, the area of event horizon can never decrease: $d A \geq 0$. 


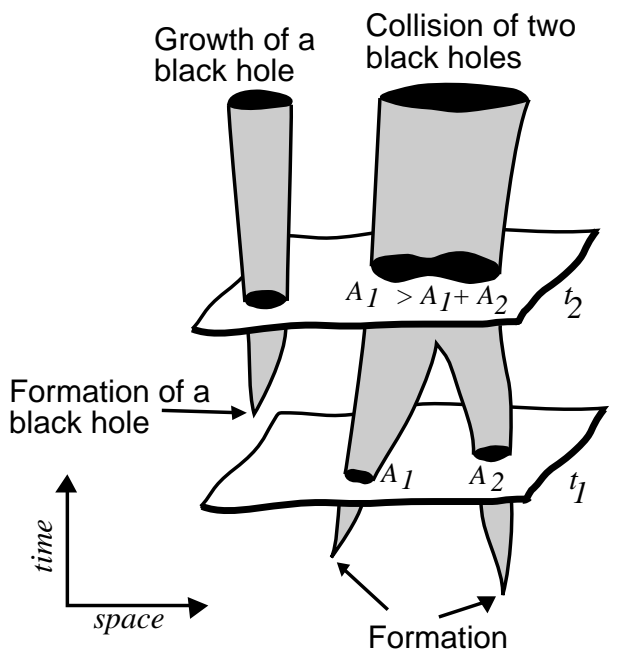

Figure 11

Fig. 11 The irreversible growth of black holes.

This law implies for instance that the area of a black hole resulting from the coalescence of two parent black holes is greater than the sum of areas of the two parent black holes (see Figure 11). It also implies that black holes cannot bifurcate, namely a single black hole can never split in two parts.

- Third law.

In thermodynamics, it reflects the inaccessibility of the absolute zero of temperature, namely it is impossible to reduce the temperature of a system to zero by a finite number of processes.

In black hole mechanics, it is impossible to reduce the surface gravity to zero by a finite number of operations. For Kerr black holes, we have seen that zero surface gravity corresponds to the "extremal" solution $J=M^{2}$.

It is clear that the area of the event horizon plays formally the role of an entropy, while the surface gravity plays the role of a temperature. However, as first pointed out by Bekenstein, if black holes had a real temperature like thermodynamical systems, they would radiate energy, contrarily to their basic definition. The puzzle was solved by Hawking when he discovered the evaporation of mini-black holes by quantum processes.

\subsection{The quantum black hole}

The details of Hawking radiation and the - not yet solved - theoretical difficulties linked to its interpretation are discussed by other lecturers (Gerard 't Hooft, 


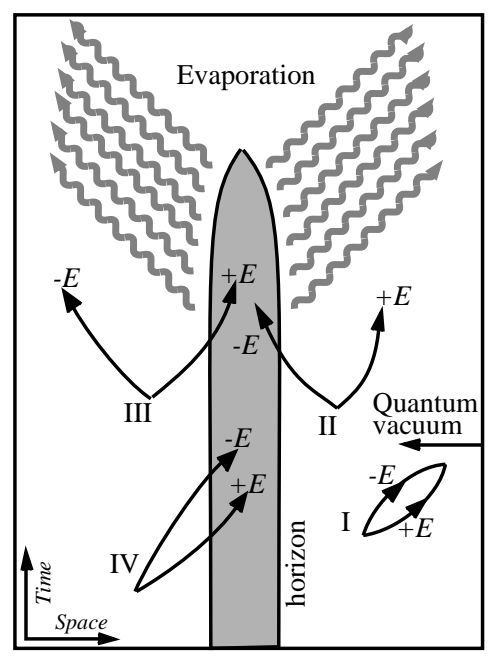

Figure 12

Fig. 12 The quantum evaporation of a mini black hole by polarisation of the vacuum.

Andreas Wipf and Claus Kiefer) in this volume. Therefore I shall only present the basic idea in a naive pictorial way (figure 12). The black hole's gravitational field is described by (classical) general relativity, while the surrounding vacuum space-time is described by quantum field theory. The quantum evaporation process is analogous to pair production in a strong magnetic field due to vacuum polarization. In the Fermi sea populated by virtual pairs of particles-antiparticles which create and annihilate themselves, the four various possible processes are depicted schematically in figure 12 .

Some virtual pairs emerging from the quantum vacuum just annihilate outside the horizon (process I). Some pairs produced in the vicinity of the black hole disappear completely in the event horizon (process IV). Some pairs are splitted, one particle (or antiparticle) escaping the black hole, the other one being captured (processes II and III). The calculations show that the process II is dominant, due to the (classical) gravitational potential which polarizes the quantum vacuum. As a consequence, a black hole radiates particles with a thermal spectrum characterized by a blackbody temperature precisely given by the formula suggested by the thermodynamical analogy:

$$
T=\hbar \frac{g}{2 \pi}=10^{-7} \frac{M_{\odot}}{M} \mathrm{~K}
$$

where $\hbar$ is Planck's constant. We immediately see that the temperature is completely negligible for any astrophysical black hole with mass comparable or greater to the solar mass. But for mini-black holes with mass $10^{15} \mathrm{~g}$ (the typ- 
ical mass of an asteroid), the Hawking temperature is $10^{12} \mathrm{~K}$. Since the black hole radiates away, it looses energy and evaporates on a timescale approximately given by

$$
t_{E} \approx 10^{10} \text { years }\left(\frac{M}{10^{15} \text { grams }}\right)^{3}
$$

Thus, mini-black holes whose mass is smaller than that of an asteroid (and size less than $10^{-13} \mathrm{~cm}$ ) evaporate on a timescale shorter than the age of the universe. Some of them should evaporate now and give rise to a huge burst of high energy radiation. Nothing similar has ever been observed ( $\gamma$-ray bursts are explained quite differently). Such an observational constraint thus limits the density of mini-black holes to be less than about $100 /(\text { lightyear })^{3}$.

The black hole entropy is given by

$$
S=\frac{k_{B}}{\hbar} \frac{A}{4}
$$

(where $k_{B}$ is Boltzmann's constant), a formula which numerically gives $S \approx$ $10^{77} k_{B}\left(\frac{M}{M_{\odot}}\right)^{2}$ for a Schwarzschild black hole. Since the entropy of a non-collapsed star like the Sun is approximately $10^{58} k_{B}$, we recover the deep meaning of the "no hair" theorem, according to which black holes are huge entropy reservoirs. By Hawking radiation, the irreducible mass, or equivalently the event horizon area of a black hole decreases, in violation of the Second Law of black hole mechanics. The latter has to be generalized to include the entropy of matter in exterior space-time. Then, the total entropy of the radiating black hole is $S=S_{B H}+S_{\text {ext }}$ and, since the Hawking radiation is thermal, $S_{\text {ext }}$ increases, so that eventually $S$ is always a non-decreasing function of time.

To conclude briefly the subject, even if mini-black holes are exceedingly rare, or even if they do not exist at all in the real universe because the big bang could not have produced such fluctuations, they represent a major theoretical advance towards a better understanding of the link between gravity and quantum theory.

\section{7 $\quad$ Space-time mappings}

Various mathematical techniques allow the geometer to properly visualize the complex space-time structure generated by black holes.

Embedding diagram - The space-time generated by a spherical mass $M$ has the Schwarzschild metric:

$$
d s^{2}=-\left(1-\frac{2 M(r)}{r}\right) d t^{2}+\left(1-\frac{2 M(r)}{r}\right)^{-1} d r^{2}+r^{2} d \Omega^{2}
$$

where $M(r)$ is the mass comprised within the radius $r$. Since the geometry is static and spherically symmetric, we do not loose much information in considering only equatorial slices $\theta=\pi / 2$ and time slices $t=$ constant. We get then a 
curved 2-geometry with metric $\left(1-\frac{2 M(r)}{r}\right)^{-1} d r^{2}+r^{2} d \phi^{2}$. Such a surface can be visualized by embedding it in Euclidean 3-space $d s^{2}=d z^{2}+d r^{2}+d \phi^{2}$. For a non-collapsed star with radius $R$, the outer solution $z(r)=\sqrt{8 M(r-2 M)}$ for $r \geq R \geq 2 M$ is asymptotically flat and matches exactly the non-singular inner solution $z(r)=\sqrt{8 M(r)(r-2 M(r))}$ for $0 \leq r \leq R$ (figure 13). For a black hole, the embedding is defined only for $r \geq 2 M$. The corresponding surface is the Flamm paraboloid $z(r)=\sqrt{8 M(r-2 M)}$. Such an asymptotically flat surface exhibits two sheets separated by the "Schwarzschild throat" of radius $2 M$. The two sheets can be either considered as two different asymptotically flat "parallel" universes (whatever the physical meaning of such a statement may be) in which a black hole in the upper sheet is connected to a time-reversed "white hole" in the lower sheet (figure 14), or as a single asymptotically flat space-time containing a pair of black/white holes connected by a so-called "wormhole" (figure 15). The freedom comes from the topological indeterminacy of general relativity, which allows us to identify some asymptotically distant points of space-time without changing the metric.

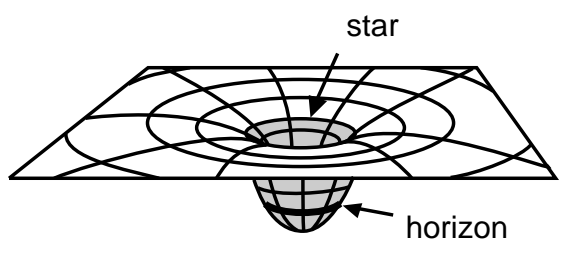

Figure 13

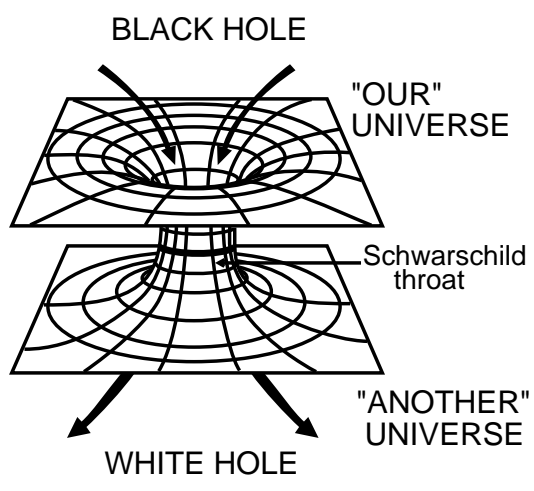

Figure 14

Fig. 13\&14 Embedding of a non-collapsed spherical star (13) and of Schwarzschild space-time (14).

However the embedding technique does not give access to the regions of space-time inside the event horizon.

Kruskal diagram - To explore inner space-time we use the maximal analytical extension of the Schwarzschild metric. This is achieved by means of a coordinate transformation discovered by Kruskal:

$$
u^{2}-v^{2}=\left(\frac{r}{2 M}-1\right) e^{r / 2 M}
$$




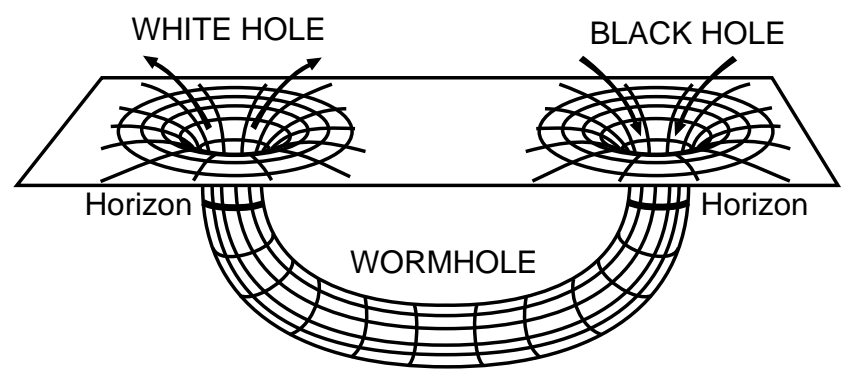

Figure 15

Fig. 15 A wormhole in space-time.

$$
\frac{v}{u}=\left\{\begin{array}{c}
\operatorname{coth} \frac{t}{4 M} \\
1 \\
\tanh \frac{t}{4 M}
\end{array}\right\} \quad \text { for } \quad r\left\{\begin{array}{l}
<2 M \\
=2 M \\
>2 M
\end{array}\right.
$$

The metric then becomes

$$
d s^{2}=\frac{32 M^{3}}{r} e^{-r / 2 M}\left(-d v^{2}+d u^{2}\right)+r^{2} d \Omega^{2}
$$

In the $(v, u)$ plane, the Kruskal space-time divides into two outer asymptotically flat regions and two regions inside the event horizon bounded by the future and the past singularities. Only the unshaded region is covered by Schwarzschild coordinates. The black region does not belong to space-time. In the Kruskal diagram (figure 16), light rays always travel at $45^{\circ}$, lines of constant distance $r$ are hyperbolas, lines of constant time $t$ pass through the origin. The interior of the future event horizon is the black hole, the interior of the past event horizon is the white hole. However it is clear that the wormhole cannot be crossed by timelike trajectories: no trajectory can pass from one exterior universe to the other one without encountering the $r=0$ singularity.

Moreover, the Kruskal extension is a mathematical idealization of a spherical black hole since it implicitly assumes that the black hole exists forever. However in the physical universe, a black hole is not inscribed in the initial conditions of the universe, it may form only from gravitational collapse. In such a case, one gets a "truncated" Kruskal diagram (figure 17), which indicates that only the future event horizon and the future singularity occur in a single asymptotically flat space-time. Such a situation offers no perspective to space-time travelers!

Penrose-Carter diagrams - The Penrose-Carter diagrams use conformal transformations of coordinates $g_{\alpha \beta} \rightarrow \Omega^{2} g_{\alpha \beta}$ which put spacelike and timelike infinities at finite distance, and thus allow to depict the full space-time into square boxes. The Penrose-Carter diagram for the Schwarzschild black hole does 


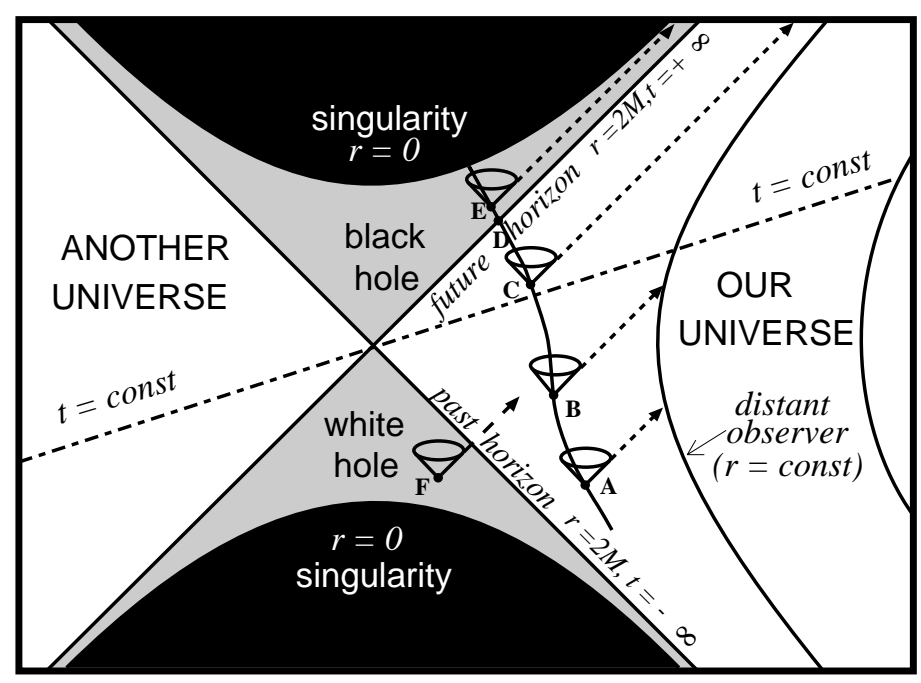

Figure 16

Fig. 16 Exploring a spherical black hole using Kruskal's map.

not bring much more information than the Kruskal one, but it turns out to be the best available tool to reveal the complex structure of a rotating black hole. Figure 18 shows the "many-fingered" universe of a Kerr black hole; it suggests that some timelike trajectories $(B, C)$ may well cross the outer $E H$ and inner $I H$ event horizons and pass from an asymptotically flat external universe to another one without encountering a singularity. This is due to the fact that the singularity $S$ is timelike rather than spacelike. Also, the shape of the singularity is a ring within the equatorial plane, so that some trajectories $(A)$ can pass through the ring and reach an asymptotically flat space-time inside the black hole where gravity is repulsive. However, the analysis of perturbations of such idealized Kerr space-times suggests that they are unstable and therefore not physically plausible. Nevertheless the study of the internal structure of black holes is a fascinating subject which is more deeply investigated in this volume by Werner Israel's lectures.

\section{Astrophysics of Black Holes}

The fact that General Relativity does predict the existence of black holes and that General Relativity is a reliable theory of gravitation does not necessarily prove the existence of black holes, because General Relativity does not describe the astrophysical processes by which a black hole may form. 


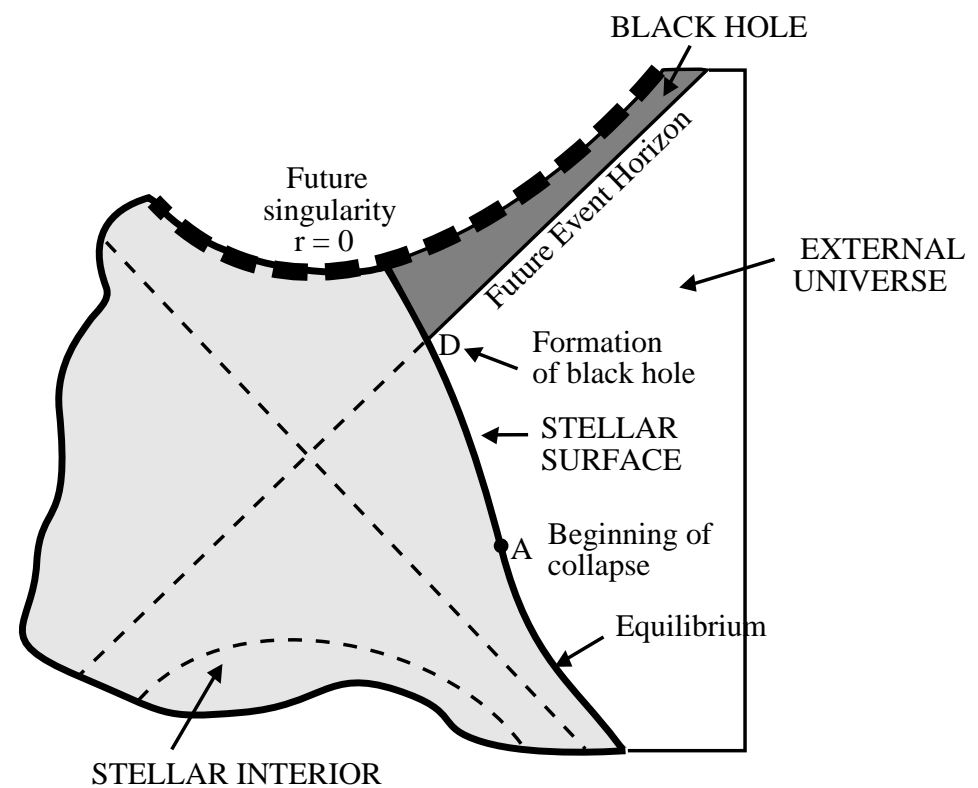

Figure 17

Fig. 17 Truncated Kruskal map representing the collapse of a star into a black hole.

Thus, the astronomical credibility of black holes crucially depends on a good understanding of gravitational collapse of stars and stellar clusters.

In this section we first examine briefly the astrophysical conditions for black hole formation, next we describe the astronomical sites where black hole candidates at various mass scales lurk.

\subsection{Formation of stellar black holes}

The basic process of stellar evolution is gravitational contraction at a rate controlled by luminosity. The key parameter is the initial mass. According to its value, stars evolve through various stages of nuclear burning and finish their lives as white dwarfs, neutron stars or black holes. Any stellar remnant (cold equilibrium configuration) more massive than about $3 M_{\odot}$ cannot be supported by degeneracy pressure and is doomed to collapse to a black hole.

The figure 19 shows the stellar paths in a density-mass diagram according to the most recent observational and theoretical data. Below $8 M_{\odot}$ stars produce white dwarfs, between 8 and $45 M_{\odot}$ they produce neutron stars; black holes are formed only when the initial mass exceeds $45 M_{\odot}$ (we note on the diagram that stars with initial mass between 20 and $40 M_{\odot}$ suffer important mass losses at 


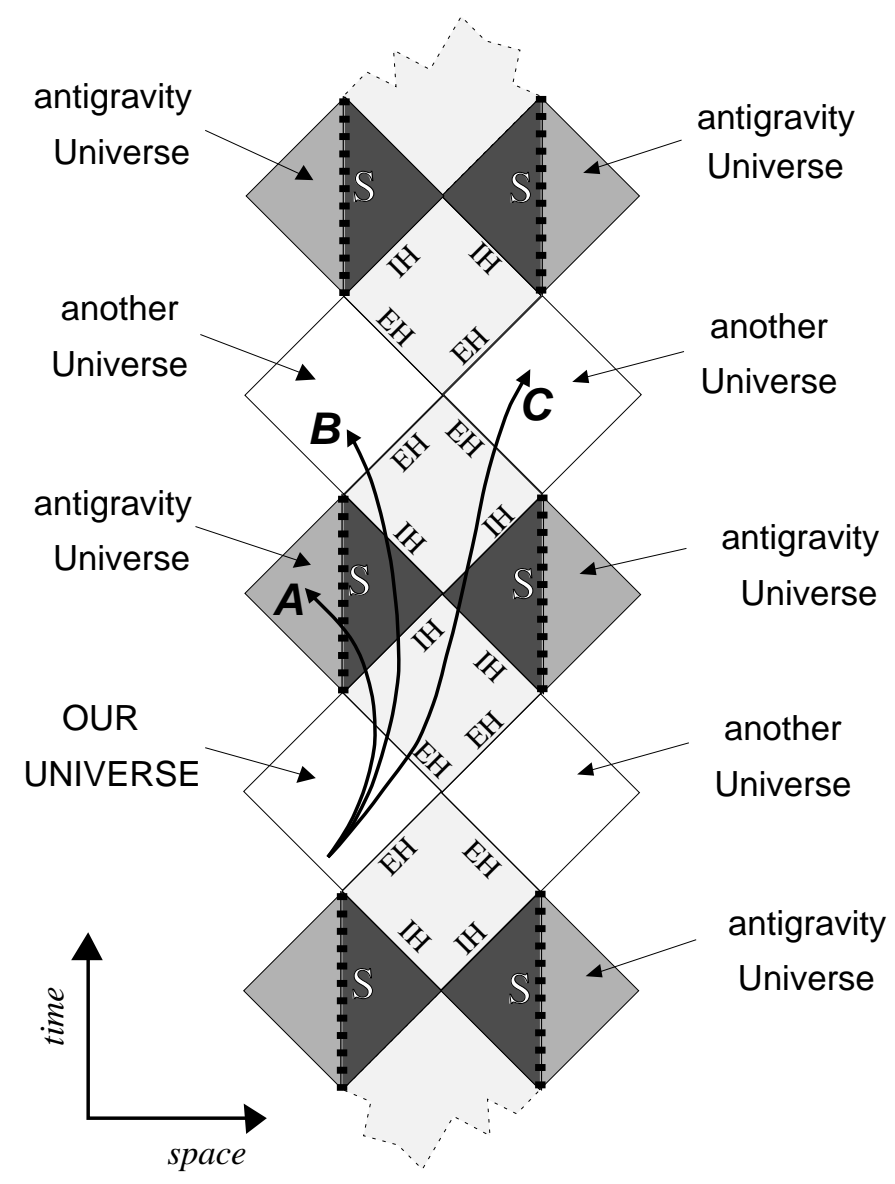

Figure 18

Fig. 18 Penrose map of a rotating black hole.

the stage of helium burning). Taking account of the stellar initial mass function, one concludes that approximately 1 supernova over 100 generates a black hole rather than a neutron star. Another possibility to form a stellar mass black hole is accretion of gas onto a neutron star in a binary system until when the mass of the neutron star reaches the maximum allowable value; then, gravitational collapse occurs and a low mass black hole forms. Taking into account these various processes, a typical galaxy like the Milky Way should harbour $10^{7}-10^{8}$ stellar black holes. 


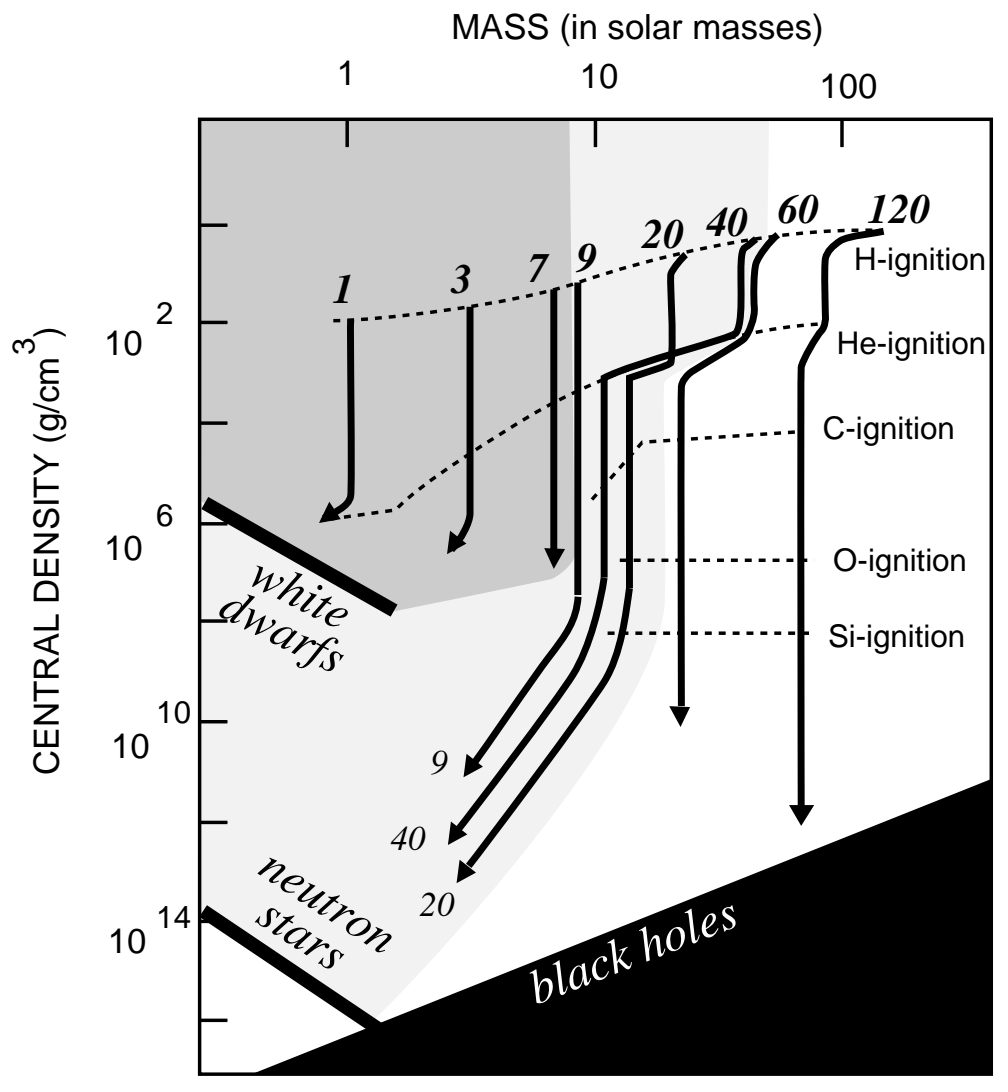

Figure 19

Fig. 19 The density-mass diagram of astronomical objects.

\subsection{Formation of giant black holes}

A massive black hole can form by continuous growth of a "seed" stellar mass black hole, by gravitational collapse of a large star cluster or by collapse of a large density fluctuation in the early universe (see next subsection). A well nourished stellar mass black hole can grow to a supermassive black hole in less than a Hubble time. Such a process requests large amounts of matter (gas and stars) in the neighborhood, a situation than can be expected in some galactic nuclei.

A dense cluster of ordinary stars, such that the velocity dispersion $v_{c} \leq v_{*}$, where $v_{*} \approx 600 \mathrm{~km} / \mathrm{s}$ is the typical escape velocity for main sequence stars, first evolves through individual stellar burning; supernovae explosions lead to the formation of compact remnants, e.g. neutron stars and stellar mass black holes. A cluster of compact stars becomes relativistically unstable at sufficiently high central gravitational redshift $1+z_{c}=(1-2 M)^{-1 / 2} \geq 1.5$ (Zeldovich 
and Podurets, 1965). Numerical simulations (e.g. Shapiro and Teukolsky, 1987, Bisnovatyi-Kogan, 1988) confirm this scenario. Starting with $\approx 10^{7}-2.10^{8}$ compact stars of $1-10 M_{\odot}$ within a cluster radius $r \leq 0.01-0.1 \mathrm{pc}$ and velocity dispersion $800-2000 \mathrm{~km} / \mathrm{s}$, the evolution proceeds through three stages:

- secular core collapse via the gravothermal catastrophe (long timescale)

- short epoch dominated by compact star collisions and coalescences, leading to the formation of black holes with mass $M \approx 90 M_{\odot}$

- relativistic instability leading to a massive black hole surrounded by a halo of stars.

\subsection{Formation of mini black holes}

Zeldovich in 1967 and Hawking in 1971 pointed out that it was in principle possible to create a black hole with small mass (e.g. below the Chandrasekhar limit) by applying a sufficiently strong external pressure. Such conditions could have been achieved only in the very early universe. Gravitational forces may locally halt the cosmic expansion of a clump of matter and reverse it into collapse if the self-gravitational potential energy of the clump exceeds the internal energy:

$$
\frac{G M^{2}}{R} \approx G \rho^{2} R^{5} \geq p R^{3}
$$

During the radiation era, $p \approx \rho c^{2}$, so the condition (17) is equivalent to $G M / c^{2} \geq$ $R$, where $R$ is the size of the fluctuation. Then a primordial black hole of mass $M$ forms. Due to the relation between density and time $G \rho \approx t^{-2}$ in an Einstein-De Sitter model of the early universe, the maximum mass of a collapsing fluctuation is related to the cosmic time by $M($ grams $) \approx 10^{38} t$ (seconds). Thus at Planck time $t \approx 10^{-43} \mathrm{~s}$, only mini black holes may form with the Planck mass $\approx 10^{-5} \mathrm{~g}$, at time $t \approx 10^{-4} \mathrm{~s}$, black holes may form with $\approx 1 M_{\odot}$, at the time of nucleosynthesis $t \approx 100 \mathrm{~s}$ supermassive black holes with $10^{7} M_{\odot}$ may form. The observational status of primordial black holes is poor and unclear. On one hand, mini black holes with mass $\leq 10^{15}$ g could be detected by a burst of $\gamma$-radiation corresponding to the last stage of quantum evaporation in less than a Hubble time. Nothing similar having been observed, this puts severe upper limits on the actual average density of mini-black holes. On the other hand, the fact that most galactic nuclei seem to harbour massive black holes (see below) and that supermassive black holes are suspected to feed quasars at very high redshift, favour the hypothesis of the rapid formation of primordial massive black holes in the early universe.

\subsection{Black Hole candidates in binary X-ray sources}

Light cannot escape (classical) black holes but one can hope to detect them indirectly by observing the electromagnetic energy released during accretion processes. 
Accretion of gas onto a compact star (neutron star or black hole in a binary system) releases energy in the X-ray domain, see S. Chakrabarti's and J. Trümper's lectures in this volume for the details. Search for stellar mass black holes thus consists in locating rapidly variable binary X-ray sources which are neither periodic (the corresponding $X$-ray pulsars are interpreted as rotating neutron stars) nor recurrent (the corresponding $X$-ray bursters are interpreted as thermonuclear explosions on a neutron star's hard surface). In spectroscopic binaries, the Doppler curve of the spectrum of the primary (visible) star provides the orbital period $P$ of the binary and the maximum velocity $v_{*}$ of the primary projected along the line-of-sight. Kepler's laws gives the following mass function which relates observed quantities to unknown masses:

$$
\frac{P v_{*}^{3}}{2 \pi G}=\frac{\left(M_{c} \sin i\right)^{3}}{\left(M_{*}+M_{c}\right)^{2}}
$$

where $M_{c}$ and $M_{*}$ are the masses of the compact star and of the optical primary, $i$ the orbital inclination angle. A crucial fact is that $M_{c}$ cannot be less than the value of the mass function (the limit would correspond to a zero-mass companion viewed at maximum inclination angle). Therefore the best black hole candidates are obtained when the observed mass function exceeds $3 M_{\odot}-$ since, according to the theory, a neutron star more massive than this limit is unstable and will collapse to form a black hole. Otherwise, additional information is necessary to deduce $M_{c}$ : the spectral type of the primary gives approximately $M_{*}$, the presence or absence of X-ray eclipses gives bounds to $\sin i$. Hence $M_{c}$ is obtained within some error bar. Black hole candidates are retained only when the lower limit exceeds $3 M_{\odot}$. At present day, about ten binary $\mathrm{X}$-ray sources provide good black hole candidates. They can be divided into two families: the highmass X-ray binaries (HMXB), where the companion star is of high mass, and the low-mass X-ray binaries (LMXB) where the companion is typically below a solar mass. The latter are also called "X-ray transients" because they flare up to high luminosities. Their mass properties are summarized in the table 1 below.

Other galactic X-ray sources are suspected to be black holes on spectroscopic or other grounds, see Chakrabarti's lectures in this volume for developments. For instance, some people argue that gamma-ray emission (above $100 \mathrm{keV}$ ) emitted from the inner edge of the accretion disc would attest the presence of a black hole rather than a neutron star, because the high-energy radiation is scattered back by the neutron star's hard surface and cools down the inner disc. If this is true, then many "gamma-ray novae" in which no measurement of mass can be done (due to the absence of optical counterpart or other limitations) are also good black hole candidates. This is specially the case for Nova Aquila 1992 and 1 E 17407-2942, two galactic sources which also exhibit radio jets. Such "microquasars" involving both accretion and ejection of matter provide an interesting link between high energy phenomena at the stellar and galactic scales, see J. Trümper's lectures. 
Table 1 Stellar mass black hole candidates

\begin{tabular}{|c|c|c|c|}
\hline & mass function & $M_{c} / M_{\odot}$ & $M_{*} / M_{\odot}$ \\
\hline$H M X B$ & & & \\
Cygnus X-1 & 0.25 & $11-21$ & $24-42$ \\
LMC X-3 & 2.3 & $5.6-7.8$ & 20 \\
LMC X-1 & 0.14 & $\geq 4$ & $4-8$ \\
\hline LMXB (X-ray transients) & & & \\
V 404 Cyg & 6.07 & $10-15$ & $\approx 0.6$ \\
A 0620-00 & 2.91 & $5-17$ & $0.2-0.7$ \\
GS 1124-68 (Nova Musc) & 3.01 & $4.2-6.5$ & $0.5-0.8$ \\
GS 2000+25 (Nova Vul 88) & 5.01 & $6-14$ & $\approx 0.7$ \\
GRO J 1655-40 & 3.24 & $4.5-6.5$ & $\approx 1.2$ \\
H 1705-25 (Nova Oph 77) & 4.65 & $5-9$ & $\approx 0.4$ \\
J 04224+32 & 1.21 & $6-14$ & $\approx 0.3-0.6$ \\
\hline
\end{tabular}

\subsection{Evidence for massive black holes in galactic nuclei}

After the original speculations of Michell and Laplace, the idea of giant black holes was reintroduced in the 1960's to explain the large amounts of energy released by active galactic nuclei (AGNs). This generic term covers a large family of galaxies including quasars, radiogalaxies, Seyfert galaxies, blazars and so on, for the classification see J. Trümper's, W. Collmar's and V. Schönfelder's lectures in this volume. The basic process is accretion of gas onto a massive black hole. The maximum luminosity for a source of mass $M$, called the Eddington luminosity, is obtained by balance between gravitational attraction and radiation pressure repulsion acting on a given element of gas. It is given by

$$
L \approx 10^{39} \frac{M}{10^{8} M_{\odot}} \mathrm{W}
$$

The observed luminosities of AGNs range from $10^{37}-10^{41} \mathrm{~W}$, where the higher values apply to the most powerful quasars. Then the corresponding masses range from $10^{6}-10^{10} M_{\odot}$.

Due to constant improvements of observational techniques, it turned out in the 1990's that most of the galactic nuclei (active or not) harbour large mass concentrations. Today the detection of such masses is one of the major goals of extragalactic astronomy. The most convincing method of detection consists in the dynamical analysis of surrounding matter: gas or stars near the invisible central mass have large dispersion velocities, which can be measured by spectroscopy. It is now likely that giant black holes lurk in almost all galactic nuclei, the energy output being governed by the available amounts of gaseous fuel. The best candidates are summarized in Table 2 . 
For instance, our Galactic Centre is observed in radio, infrared, X-ray and gamma-ray wavelengths (other wavelengths are absorbed by dust clouds of the galactic disc). A unusual radiosource has long been observed at the dynamical centre, which can be interpreted as low-level accretion onto a moderately massive black hole. However, a definite proof is not yet reached because gas motions are hard to interpret. Recently Eckart and Genzel (1996) obtained a full threedimensional map of the stellar velocities within the central $0.1 \mathrm{pc}$ of our Galaxy. The values and distribution of stellar velocities are convincingly consistent with the hypothesis of a $2.5 \times 10^{6} M_{\odot}$ black hole.

The nucleus of the giant elliptical M87 in the nearby Virgo cluster has also a long story as a supermassive black hole candidate. Several independent observations are consistent with a $1-310^{9} M_{\odot}$ black hole accreting in a slow, inefficient mode. A disc of gas is orbiting in a plane perpendicular to a spectacular jet; recent spectroscopic observations of the Hubble Space Telescope show redshifted and blueshifted components of the disc, which can be interpreted by Doppler effect as parts of the disc on each side of the hole are receding and approaching from us.

The spiral galaxy NGC 4258 (M 106) is by far the best massive black hole candidate. Gas motions near the centre has been precisely mapped with the 1.3 $\mathrm{cm}$ maser emission line of $\mathrm{H}_{2} \mathrm{O}$. The velocities are measured with accuracy of 1 $\mathrm{km} / \mathrm{s}$. Their spatial distribution reveals a disc with rotational velocities following an exact Kepler's law around a massive compact object. Also the inner edge of the disc, orbiting at $1080 \mathrm{~km} / \mathrm{s}$, cannot comprise a stable stellar cluster with the inferred mass of $3.6 \times 10^{7} M_{\odot}$.

Table 2 Massive black hole candidates

\begin{tabular}{|c|c|c|c|}
\hline dynamics & host galaxy & galaxy type & $M_{h} / M_{\odot}$ \\
\hline maser & M 106 & barred & $4 \times 10^{7}$ \\
gas & M 87 & elliptical & $3 \times 10^{9}$ \\
gas & M 84 & elliptical & $3 \times 10^{8}$ \\
gas & NGC 4261 & elliptical & $5 \times 10^{8}$ \\
\hline stars & M 31 & spiral & $3-10 \times 10^{7}$ \\
stars & M 32 & elliptical & $3 \times 10^{6}$ \\
stars & M 104 & (barred?) spiral & $5-10 \times 10^{8}$ \\
stars & NGC 3115 & lenticular & $7-20 \times 10^{8}$ \\
stars & NGC 3377 & elliptical & $8 \times 10^{7}$ \\
stars & NGC 3379 & elliptical & $5 \times 10^{7}$ \\
stars & NGC 4486B & elliptical & $5 \times 10^{8}$ \\
stars & Milky Way & spiral & $2.5 \times 10^{6}$ \\
\hline
\end{tabular}


The black hole in our galaxy and the massive black holes suspected in nearby ordinary galaxies would be small scale versions of the cataclysmic phenomena occurring in AGNs. But AGNs are too far away to offer a spectral resolution good enough for dynamical measurements. Indeed, estimates of luminosities of AGNs and theoretical arguments involving the efficiency of energy release in strong gravitational fields invariably suggest that central dark masses are comprised between $10^{7}-10^{9} M_{\odot}$. Variability of the flux on short timescales also indicates that the emitting region has a small size; many AGNs exhibit large luminosity fluctuations over timescales as short as one hour, which indicate that the emitting region is smaller than one light-hour. Such large masses in such small volumes cannot be explained by star clusters, so that accreting massive black holes remain the only plausible explanation.

\subsection{Stellar disruption}

The accretion of gas at rate $d M / d t$ and typical efficiency $\epsilon \approx 0.1$ produces a luminosity

$$
L \approx 10^{39}\left(\frac{\epsilon}{0.1}\right) \frac{d M / d t}{1 M_{\odot} / \text { year }} \mathrm{W}
$$

By comparing the luminosity of the accretion model with the observed luminosities in AGNs, we conclude that the gas accretion rate must lie in the range $10^{-2}-10^{2} M_{\odot} /$ year. One is thus led to the question about the various gas production mechanisms able to fuel a giant black hole. An efficient process is mass loss from stars passing near the hole. Current models of galactic nuclei involve a massive black hole surrounded by a dense large cloud of stars. Diffusion of orbits makes some stars to penetrate deeply within the gravitational potential of the black hole along eccentric orbits. Disruption of stars can occur either by tidal forces or by high-velocity interstellar collisions (figure 20). The collision radius $R_{\text {coll }} \approx 7 \times 10^{18} \frac{M}{10^{8} M_{\odot}} \mathrm{cm}$ for a solar-type star is the distance within which the free-fall velocity of stars becomes greater than the escape velocity at the star's surface $v_{*}$ (typically $500 \mathrm{~km} / \mathrm{s}$ for ordinary stars); if two stars collide inside $R_{\text {coll }}$ they will be partially or totally disrupted.

Also stars penetrating the critical tidal radius $R_{T} \approx 6 \times 10^{13}\left(\frac{M}{10^{8} M_{\odot}}\right)^{1 / 3} \mathrm{~cm}$ for solar-type stars will be ultimately disrupted, about 50 per cent of the released gas will remain bound to the black hole. In some sense, the tidal encounter of a star with a black hole can be considered as a collision of the star with itself...

In the collision process the factor $\beta=v_{\text {rel }} / v_{*}$ plays a role analogous to the penetration factor $\beta=R_{T} / R_{p}$ in the tidal case (where $R_{p}$ is the periastron distance). As soon as $\beta \geq 1$ the stars are disrupted, and when $\beta \geq 5$ the stars are strongly compressed during the encounter. Thus, in both cases, $\beta$ appears as a crushing factor, whose magnitude dictates the degree of maximal compression and heating of the star.

The first modelisation of the tidal disruption of stars by a big black hole was done in the 1980's by myself and collaborators (see Luminet and Carter, 1986 

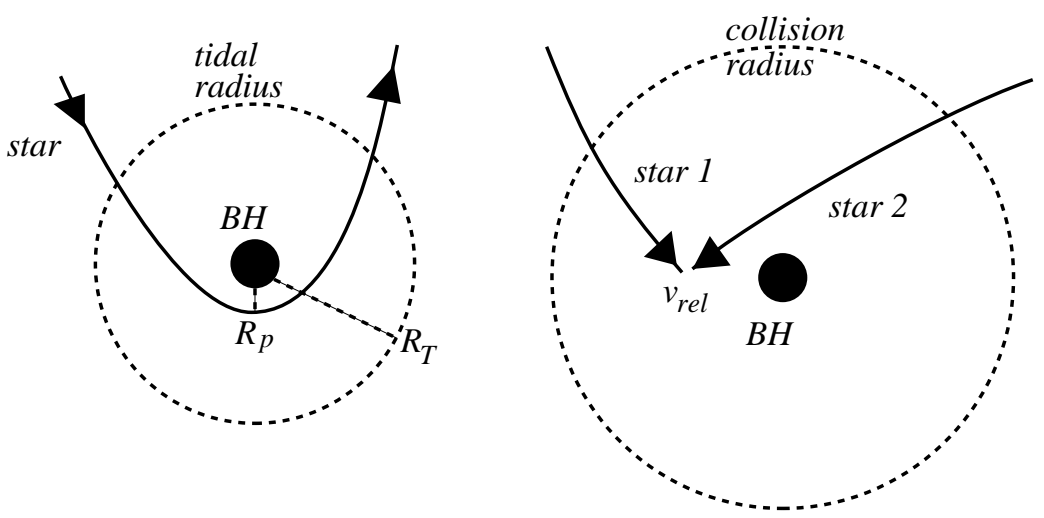

Figure 20

Fig. 20 Tidal and collision radius.

and references therein). We have discovered that a star deeply plunging in the tidal radius without crossing the event horizon is squeezed by huge tidal forces and compressed into a short-lived, ultra-hot "pancake" configuration. Figure 21 shows schematically the progressive deformation of the star (the size of the star has been considerably over-emphasized for clarity). The left diagram represents the deformation of the star in its orbital plane (seen from above), the right one shows the deformation in the perpendicular direction. From $a$ to $d$ the tidal forces are weak and the star remains practically spherical. At $e$ the star penetrates the tidal limit. It becomes cigar-shaped. From $e$ to $g$ a "mangle" effect due to tidal forces becomes increasingly important and the star is flattened in its orbital plane to the shape of a curved "pancake". The star rebounds, and as it leaves the tidal radius, it starts to expand, becoming more cigar-shaped again. A little further along its orbit the star eventually breaks up into fragments.

If the star chances to penetrate deeply (says with $\beta \geq 10$ ), its central temperature increases to a billion degrees in a tenth of a second. The thermonuclear chain reactions are considerably enhanced. During this brief period of heating, elements like helium, nitrogen and oxygen are instantaneously transformed into heavier ones by rapid proton or alpha-captures. A thermonuclear explosion takes place in the stellar pancake, resulting in a kind of "accidental supernova". The consequences of such an explosion are far reaching. About 50 per cent of the stellar debris is blown away from the black hole at high velocity (propelled by thermonuclear energy release), as a hot cloud able of carrying away any other clouds it might collide with. The rest of the debris falls rapidly towards the hole, producing a burst of radiation. Like supernovae, the stellar pancakes are also crucibles in which heavy elements are produced and then scattered throughout the galaxy. Thus, observation of high-velocity clouds and enrichment of the interstellar medium by specific isotopes in the vicinity of galactic nuclei would 


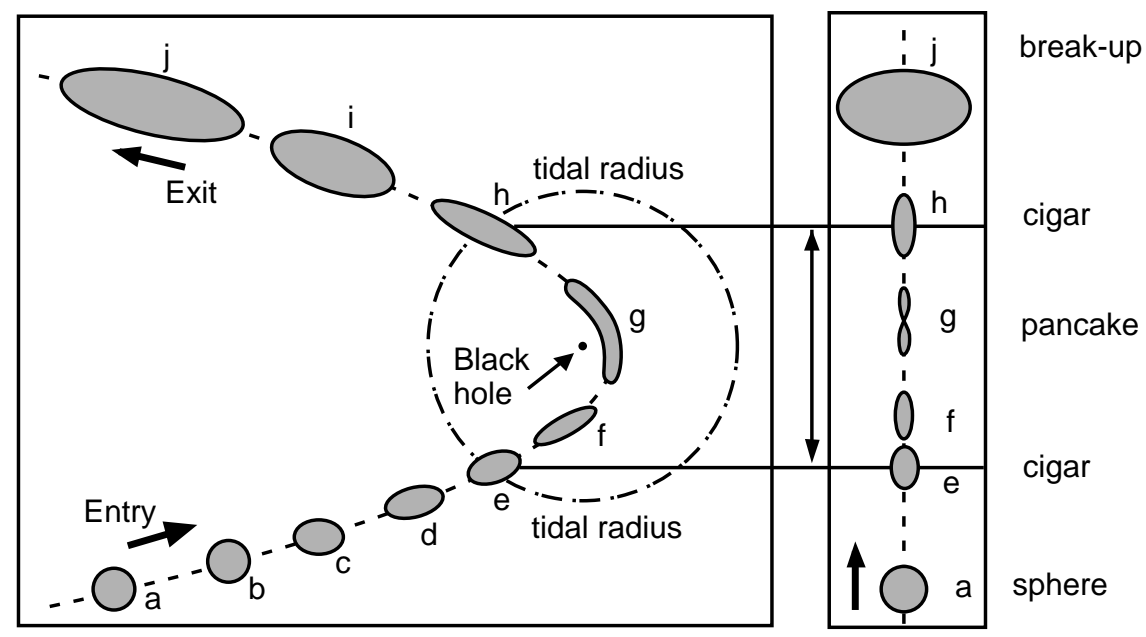

Figure 21

Fig. 21 The disruption of a star by tidal forces of a black hole.

constitute an observational signature of the presence of big black holes.

Explosive or not, the tidal disruption process would induce a burst of luminosity in the host galactic nucleus on a timescale of a few months (the time required for the debris to be digested). To describe the evolution of the star we developed a simplified "affine model" in which we assumed that the layers of constant density keep an ellipsoidal form. Many astrophysicists were skeptical about the predictions of the model until when full hydrodynamical calculations were performed all around the world, using 3D Smooth Particle Hydrodynamical codes (Laguna and Miller, 1993, Khlokov, Novikov and Pethick, 1993, Frolov et al., 1994) or spectral methods (Marck et al., 1997). The main features and quantitative predictions of the affine star model were confirmed, even if shock waves may decrease a little bit the maximum pancake density.

The nucleus of the elliptical galaxy NGC 4552 has increased its ultra-violet luminosity up to $10^{6} L_{\odot}$ between 1991 and 1993 (Renzini et al, 1993). The timescale was consistent with a tidal disruption process, however the luminosity was $\approx 10^{-4}$ lower than expected, suggesting only a partial disruption of the star.

\section{A journey into a black hole}

Imagine a black hole surrounded by a bright disc (Figure 22). The system is observed from a great distance at an angle of $10^{\circ}$ above the plane of the disc. The light rays are received on a photographic plate (rather a bolometer in order to capture all wavelengths). Because of the curvature of space-time in the 


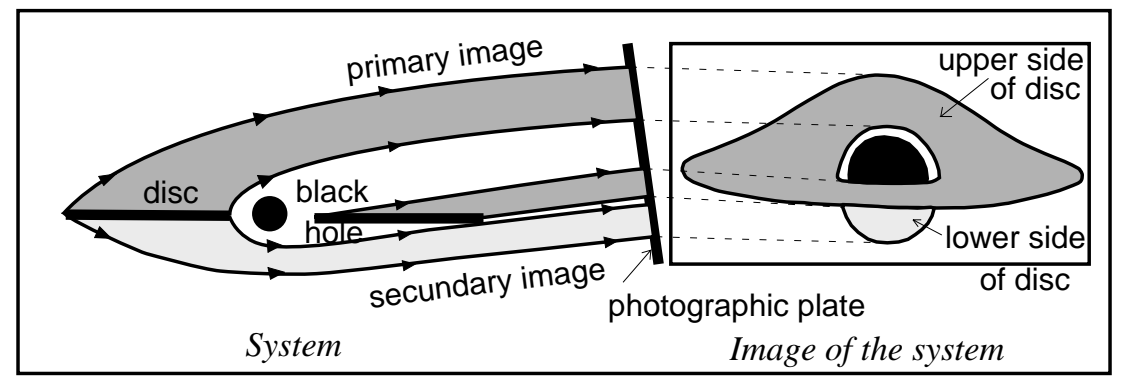

Figure 22

Fig. 22 Optical distortions near a black hole.

neighborhood of the black hole, the image of the system is very different from the ellipses which would be observed if an ordinary celestial body (like the planet Saturn) replaced the black hole. The light emitted from the upper side of the disc forms a direct image and is considerably distorted, so that it is completely visible. There is no hidden part. The lower side of the disc is also visible as an indirect image, caused by highly curved light rays.

The first computer images of the appearance of a black hole surrounded by an accretion disc were obtained by myself (Luminet, 1978). More sophisticated calculations were performed by Marck (1993) in Schwarzschild and Kerr spacetimes. A realistic image, e.g. taking account of the space-time curvature, of the blue- and redshift effects, of the physical properties of the disc and so on, can be precisely calculated at any point of space-time - including inside the event horizon. A movie showing the distortions observed along any timelike trajectory around a black hole was produced (Delesalle, Lachièze-Rey and Luminet, 1993). The figure 23 is a snapshot taken along a parabolic plunging trajectory. During such a "thought journey" the vision of the third butterfly becomes accessible, all external spectators can admire the fantastic landscape generated by the black hole.

For a long time considered by astronomers as a mere theoretical speculation, black holes are now widely accepted as the basic explanation for X-ray massive binaries and galactic nuclei. Allowing for the elaboration of the most likely models, black holes also respond to the principle of simplicity, according to which among equally plausible models, the model involving the least number of hypotheses must be preferred. However, for such a wide acceptance to be settled down, the basic picture of a black hole had to be drastically changed. The conjunction of theoretical and observational investigations allowed for such a metamorphosis of the black hole image, passing from the primeval image of a naked black hole perfectly passive and invisible, to the more sophisticated image of a thermodynamical engine well-feeded in gas and stars, which turns out to 


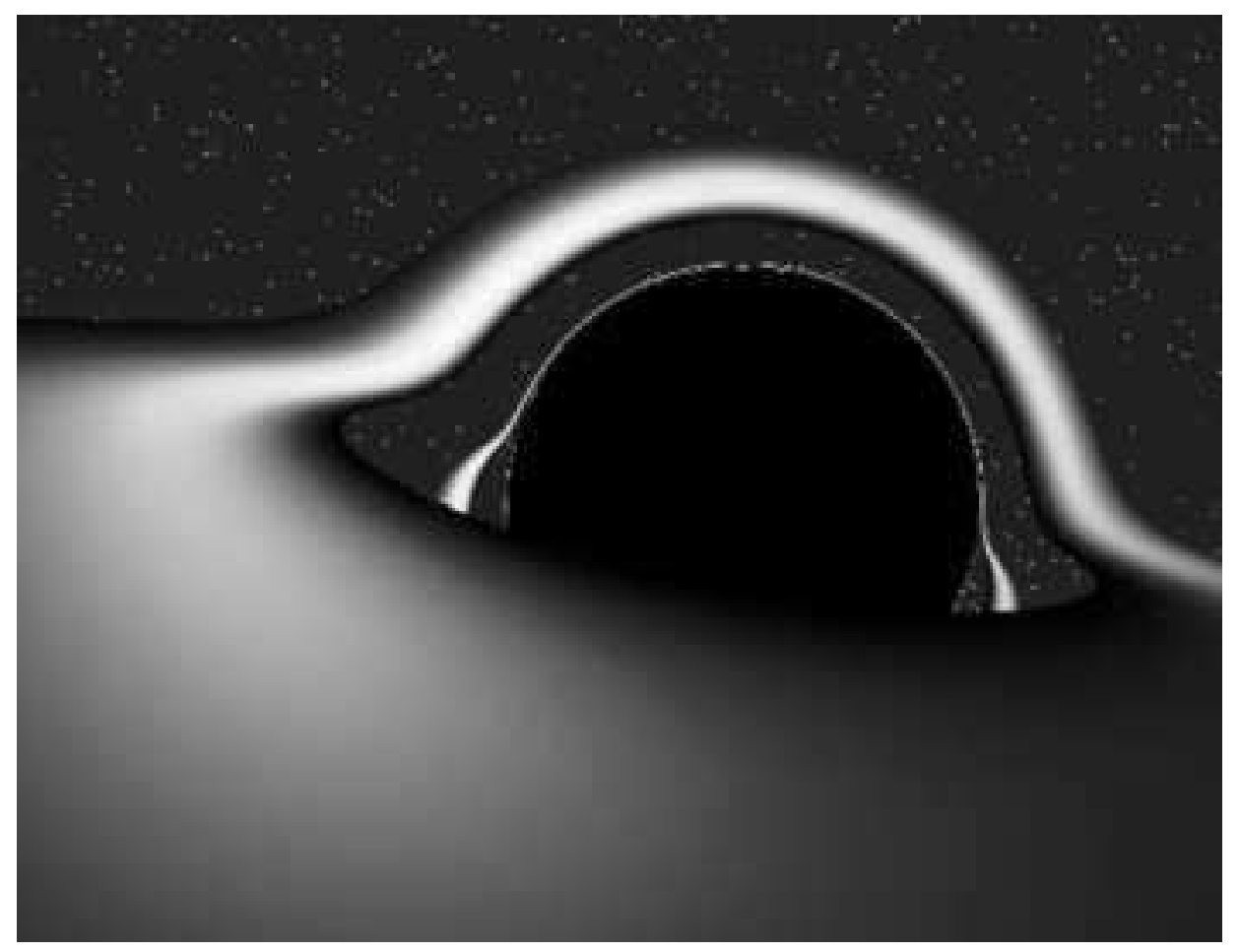

Fig. 23 The appearance of a distant black hole surrounded by an accretion disc. (C)J.-A.Marck/Sygma)

be the key of the most luminous phenomena in the universe. Then the modern astronomer won over to such a duality between light and darkness may adopt the verse of the french poet Léon Dierx: "Il est des gouffres noirs dont les bords sont charmants".

\section{References}

[1] Begelman, M., Rees, M. (1996): Gravity's Fatal Attraction: Black Holes in the Universe (New York: Scientific American Library)

[2] Chandrasekhar, S. (1992): The Mathematical Theory of Black Holes, (Oxford: Oxford University Press)

[3] Delesalle, L., Lachièze-Rey, M., Luminet J.-P. (1994): Infinitely Curved, video VHS $52 \mathrm{mn}$, Arte/CNRS Audiovisuel

[4] DeWitt, C., DeWitt, B.S. (Eds.) (1973): Black Holes (Les Houches School, Gordon and Breach: New York) 
[5] Hawking, S.W., Israel, W. (Eds.) (1989): 300 Years of Gravitation (New York, Cambridge: Cambridge University Press), pp. 199-446

[6] Luminet, J.-P. (1979): Astron. Astrophys. 75, 228

[7] Luminet, J.-P., Carter, B. (1986): Astrophys. J. Suppl. 61, 219

[8] Luminet, J.-P. (1992): Black Holes (New York, Cambridge: Cambridge University Press). German translation: Schwarze Löcher (Vieweg, 1997)

[9] Marck, J.-A. (1993): Class. Quantum Grav. 13, 393

[10] Misner, C.W., Thorne, K.S., Wheeler, J.A. (1973): Gravitation (San Francisco: Freeman)

[11] O'Neill, B. (1995): The Geometry of Kerr Black Holes (Wellesley: Peters)

[12] Wheeler, J.A. (1990): A Journey into Gravity and Spacetime. (New York, Cambridge: Cambridge University Press) 


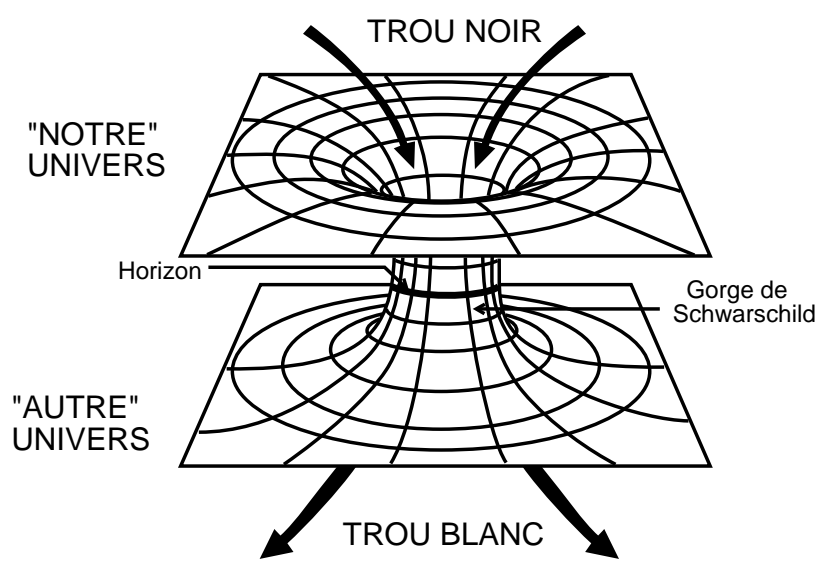

